\title{
EMF of Concentration Cells with Liquid Junction Established by Free Diffusion. Part II. Applicability of Some Theoretical Models on Experimental Results for $\mathrm{BaCl}_{2} / \mathbf{H C l}$ Junctions
}

\author{
NIELS OLAF ØSTERBERG, ${ }^{a}$ JØRGEN BIRGER JENSEN, ${ }^{a}{ }^{\text {TORBEN SMITH SØRENSEN }}{ }^{\mathrm{b}}$ and \\ LEIF DYHRBERG CASPERSEN ${ }^{a}$
}

${ }^{a}$ Fysisk-Kemisk Institut, DTH 206, DK-2800 Lyngby, Denmark and b Fysisk Laboratorium III, DTH 309, DK-2800 Lyngby, Denmark

Emf's have been measured at $25^{\circ} \mathrm{C}$ on the following cell:

$\mathrm{Pt}|\mathrm{Ag}| \mathrm{AgCl}\left|\mathrm{BaCl}_{2}\left(c_{1}\right)\right| \mathrm{HCl}\left(c_{2}\right)|\mathrm{AgCl}| \mathrm{Ag} \mid \mathrm{Pt}$ $c_{1} \in\left[5 \times 10^{-4} \mid 1.0\right], c_{2} \in\left[1 \times 10^{-3} \mid 0.1\right]$,

$c$ in $\mathrm{mol} \mathrm{dm}^{-3}$.

The liquid junction between the two half cells is established as free diffusion under carefully controlled experimental conditions.

The results have been statistically treated by means of a polynomium fitting program in order to improve the experimental precision.

The smoothened results are compared with the simple Henderson equation and with some modified versions of the Henderson formula. Furthermore, comparison is made with the Planck-Pleijel model. The simple Planck-Pleijel model seems better than the simple Henderson model, but in both cases an asymmetry exists due to the use of mobilities at infinite dilution. The best of the investigated models is a modified Henderson model using concentration dependent conductivities and transport numbers (predictive precision around $\pm 7 \mathrm{mV}$ ). The total range of measured emf-values spans about $300 \mathrm{mV}$.

When two electrolyte solutions of different concentration and/or electrolyte are brought into internal electrical and chemical contact, a liquidliquid junction potential is created. This junction potential contributes to the total emf measured in electrochemical cells.

Emf-measurements on such cells can be carried out with great accuracy, and under well-defined experimental conditions it is possible to obtain reproducible results which agree within $\pm 0.3 \mathrm{mV}$.

Contrary to the total emf which can be expressed through salt activities and transport numbers, it is not possible to determine the exact contribution from the liquid junction potential. The reason for this is that single ion activties cannot be determined by traditional electrochemical means. Also, it is troublesome to determine the total emf in cells with more than one diffusing electrolyte, because of the lack of knowledge of the concentration profiles and the lack of proper experimental values for transference numbers and salt activity coefficients in electrolyte mixtures.

In order to estimate the junction potential we are forced to apply theoretical models in connection with assumptions concerning the splitting of salt or mean ionic activity coefficients into single ion activity coefficients. Henderson ${ }^{1}$ and Pleijel ${ }^{2}$ have derived such models based on different assumptions. The Pleijel model is a generalisation of an earlier model proposed by Planck ${ }^{3}$ to other electrolytes than uni-uni valent ones.

Those three models have been modified in different directions by a number of authors. Lewis and Sargent ${ }^{4}$ consider the special case of two uni-uni valent electrolytes of equal concentration with the junction potential given by eqn. (1). The $\Lambda$ 's are

$\Delta \phi \equiv \phi$ r.h.s. $-\phi$ l.h.s. $=\frac{R T}{F} \ln \frac{\Lambda(\text { l.h.s. })}{\Lambda \text { (r.h.s. })}$

equivalent or molar conductivities for the two salts. Formula (1) can be derived from the Henderson 
formula (continuous mixture zone) as well as from the Planck-Pleijel model (steady state diffusion in a finite layer), see, e.g. Ref. 5 pp. 233-234.

Strictly speaking, the equivalent conductivities in (1) should be taken at infinite dilution, but Lewis and Sargent obtain some correction by using instead the actual conductivities in the electrode solutions. Spiro ${ }^{6}$ has extended the Lewis and Sargent case by correcting also for activity coefficients in a continuous mixture zone.

Bass ${ }^{7}$ has relaxed the electroneutrality condition in Planck's model. He finds the potential difference of Planck to be the first term in an asymptotic expansion and he calculates the second term. Deviation from electroneutrality becomes important only for diffusion zones below about $1000 \AA$ in thickness.

Goldman $^{8}$ has involved an entirely different assumption of constant electric field through the zone or membrane. The Goldman model has become popular among nerve and cell physiologists. The assumption is not a good one, however, since the electric field is far from constant in a diffusion zone; see for example the numeric calculations of Sørensen and Jensen. ${ }^{9}$ Also, in the case of diffusion of a single uni-uni valent electrolyte the model differs from the result of the Planck model as shown by Goldman himself [Ref. 8, p. 53, eqns. (13) and (15)]. Since the result obtained from the Planck model is the same as that obtained from Henderson's model and from simple irreversible thermodynamics in that particular case, we are obliged to reject the Goldman formula as also done by Zelman ${ }^{10}$ on different grounds.

The experimental tests of the different theoretical models have until now been restricted to uni-uni valent electrolytes and mostly to the $\mathrm{KCl}-\mathrm{HCl}$ system. In a precursor to the present paper, ${ }^{11}$ however, the $\mathrm{BaCl}_{2}-\mathrm{HCl}$ system was studied by emfmeasurements on the cell (2), where $C_{1}$ and $C_{2}$ are

$$
\mathrm{Pt}|\mathrm{Ag}| \mathrm{AgCl}\left|\mathrm{BaCl}_{2}\left(C_{1}\right): \mathrm{HCl}\left(C_{2}\right)\right| \mathrm{AgCl}|\mathrm{Ag}| \mathrm{Pt}
$$

concentrations in $\mathrm{mol} \mathrm{dm} \mathrm{dm}^{-3}$ in the intervals $\left(0.5 \times 10^{-3} \mid 1.0\right)$ and $\left(1 \times 10^{-3} \mid 0.1\right)$, respectively. In the preceding paper only the simple Henderson model was tested and relatively few experiments were considered.

In the present paper a much better statistical material is available. About 175 experiments have been carried out on the cell (2) in almost the same ranges of concentration, but with improved experimental technique. The statistical smoothening is made by a polynomium fitting program ${ }^{12}$ and new, extended Henderson and Pleijel models are included in the comparisons.

\section{EXPERIMENTAL}

Chemicals. $\mathrm{HCl}, \mathrm{BaCl}_{2}$ and $\mathrm{KAg}(\mathrm{CN})_{2}$ solutions were prepared as described previously. ${ }^{11}$

Apparatus. The experiments reported in part $\mathrm{I}^{11}$ were carried out in a water-jacketed diffusion column with circular cross section with an inside diameter of $8 \mathrm{~mm}$. In that diffusion column the length of the diffusion zone could be max. $140 \mathrm{~mm}$ which corresponds to the distance between the two $\mathrm{Ag} / \mathrm{AgCl}$-electrodes. However, during these experiments it was found desirable with a somewhat longer diffusion zone in order to prolong the duration of the period with stable potential. In con-

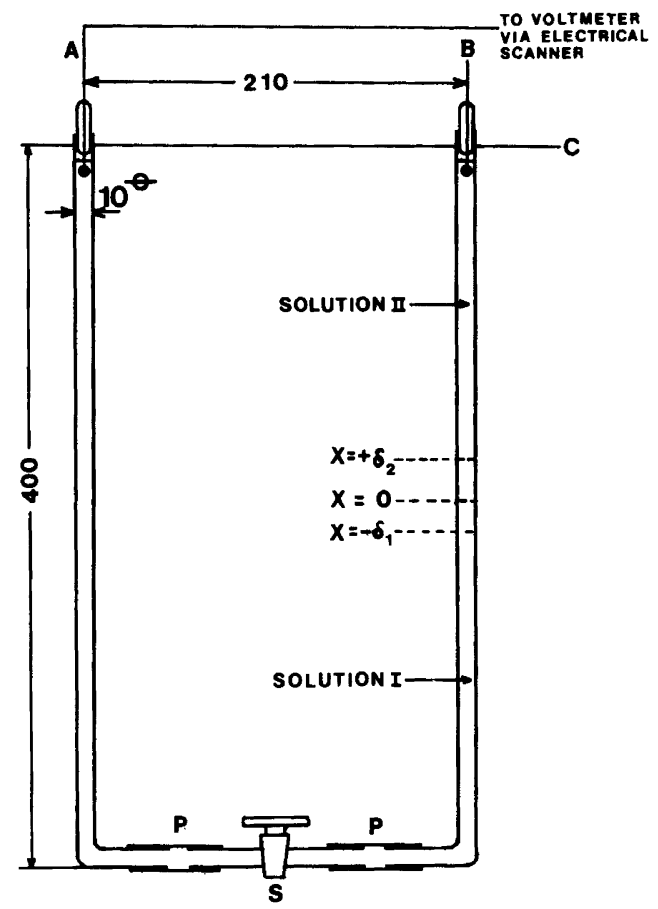

Fig. 1. Diffusion column. Dimensions in $\mathrm{mm}$ (approximate values). The free diffusion junction established at $x=0$. Distance from $x=-\delta_{1}$ to $x=+\delta_{2}$ indicates length of diffusion zone at the time $t=\tau$. Emf is measured between the two $\mathrm{Pt}|\mathrm{Ag}| \mathrm{AgCl}-\mathrm{electrodes} \mathrm{A}$ and B. Level of thermostat water is indicated by C. S: stopcock for interconnection of the two cylindrical glass tubes. P: plastic tubes. 


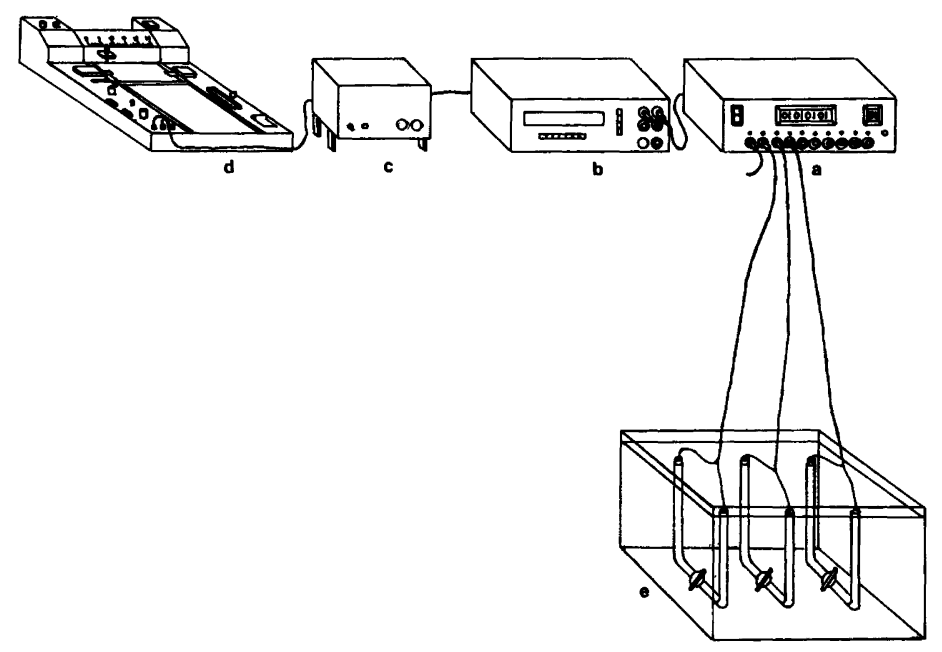

Fig. 2. Diagram of experiment. a, electrical scanner (Multiplexer). b, digital voltmeter. c, BCD-ANALOG converter. d, recorder. e, water-thermostat with three diffusion columns. (Supporting stands omitted for the sake of clarity.).

sequence, a new diffusion column was built up from two L-shaped glass tubes interconnected via a screw cock and plastic tubes.

The general feature of the diffusion column is outlined in Fig. 1. Three such diffusion columns were founded on a common plate of plexiglass with a distance of about $100 \mathrm{~mm}$ and simultaneously immersed in a water bath $\left(25.0 \pm 0.1^{\circ} \mathrm{C}\right)$. With such arrangements a stable potential could be observed for several hours. The potential stability during this period was $\pm 10 \mu \mathrm{V}$.

Measuring devices. The emf-measurements were performed on a $4 \frac{1}{2}$ digital multimeter from Data Precision, Wakefield, Mass., USA. With this instrument it is possible to measure emf below $200 \mathrm{mV}$ with an accuracy of $\pm 10 \mu \mathrm{V}$. By means of a BCDANALOG converter, made by Ring Instrument, Vanløse, Denmark, a Radiometer recorder, REC61, was connected to the multimeter and the last three digits were recorded $(1 \mathrm{mV} \sim 1$ volt output). The BCD-ANALOG converter was optically connected in order to avoid electrical interference between recorder and multimeter. An electrical scanner ("Multiplexer") made at Fysisk-Kemisk Institute and placed between the diffusion column and the multimeter makes it possible to measure and record successively nine different diffusion columns. The measuring devices connected to three diffusion columns are schematically shown in Fig. 2. One of the connections to the electrical scanner is shortcircuited in order currently to be able to control the electrical stability of the experimental equipment.
Silver-silver chloride electrodes. Two types of silver-silver chloride electrodes were used during the experiments: (1) An electrolytic type prepared as described earlier ${ }^{11}$ and (2) a thermal-electrolytic type prepared and kindly delivered from Radiometer A/S, Copenhagen, Denmark. The preparation procedure of the latter type is shortly mentioned in the books of Bates ${ }^{13}$ and Ives and Janz. ${ }^{14}$ From comparison of the two types of electrodes we made the following main observations:

(1) The equilibration periods were remarkably shorter on the thermo-electrolytically prepared $\mathrm{Ag} / \mathrm{AgCl}$ electrodes than on the electrolytically prepared ones.

(2) The reproducibility of the asymmetry potentials was considerably better for the thermoelectrolytically prepared $\mathrm{Ag} / \mathrm{AgCl}$ electrodes. However, in no case was an electrode pair used in the experiments with asymmetry potential greater than $\pm 0.1 \mathrm{mV}$.

(3) No light effect was observed on the thermoelectrolytically prepared $\mathrm{Ag} / \mathrm{AgCl}$ electrode used in these experiments.

Measuring technique. Before each experiment the diffusion column was thoroughly rinsed several times with distilled and deionized water followed by a two times wash with absolute ethyl alcohol. Finally, the diffusion column was dried under suction from a water pump for at least one hour. Then the more dense solution was filled into the column through the left-hand glass tube up to the middle of the tubes. The screw-cock was closed and the rest 
of the glass tube on the left-hand side was filled up with the same solution. By means of an automatic burette (ABU 12 from Radiometer) adjusted at the beginning to give a flow of $1.9 \mathrm{ml} \mathrm{h}^{-1}$ the second solution was very carefully placed on top of the first one in the right-hand glass tube. In this way the liquid junction could be formed with a sharp boundary, as could sometimes be observed by means of the difference in the refraction index of the solutions. When the surface of the two solutions was completely level, the $\mathrm{Ag} / \mathrm{AgCl}$ electrodes were carefully placed on top of the tubes and immersed into the solutions. (Height levels due to differences in densities of the two solutions turned out to be so small that they could be ignored.) The screw-cock was opened and emf-measurements were started and continued during a period of at least 24 hours for each experiment. During such measurement a period with stable potential could be observed for $10-15 \mathrm{~h}$. The stability of the asymmetric potential was controlled before and after each experiment. Further, before each experiment, the electrodes were allowed to equilibrate at least one hour in the solutions applied for the measurements.

Measurements have been made with the following fixed $\mathrm{HCl}$-concentrations: $0.1,0.01,0.001$, and 0.0001 and varying concentrations of $\mathrm{BaCl}_{2}$ between $5 \times 10^{-4}$ and 1.0 (all concentrations in mol $\mathrm{dm}^{-3}$ ). For practical reasons, measurements involving one $\mathrm{HCl}$-concentration were finished before a new $\mathrm{HCl}$-concentration was introduced. The advantage of this procedure was that the autoburette could continuously be filled with the same concentration of $\mathrm{HCl}$ during a longer period and thus the risk of mixing the electrolytes was minimized.

\section{THEORY}

In the model of Henderson ${ }^{1}$ a continuous mixture zone is assumed between the two electrolyte solutions, i.e. for the concentration of the i'th ionic species at any place in the diffusion zone eqn. (3)

$C_{\mathrm{i}}(\mathrm{x})=C_{\mathrm{i}}(\mathrm{I})+\alpha(\mathrm{x}) \Delta C_{\mathrm{i}}$

is assumed, with the relation (4) and with $\alpha$ as a

$\Delta C_{\mathrm{i}}=C_{\mathrm{i}}(\mathrm{II})-C_{\mathrm{i}}(\mathrm{I})$

parameter varying continuously from 0 in solution I to 1 in solution II. Under this assumption and applying eqns. (3) and (4) together with the assumptions of ideality, i.e. (1) ionic activities equal to ionic concentrations, (2) constant and independent ionic mobilities $U_{\mathfrak{i}}$, the expression for the diffusion potential can immediately be integrated to

$\Delta \phi_{\mathrm{H}}=-\frac{R T}{F} \frac{\sum_{z_{\mathrm{i}}} U_{\mathrm{i}} \Delta C_{\mathrm{i}}}{\sum z_{k}^{2} U_{k} \Delta C_{k}} \ln \frac{\Sigma z_{\mathrm{i}}^{2} U_{\mathrm{i}} C_{\mathrm{i}}(\mathrm{II})}{\sum z_{k}^{2} U_{k} C_{k}(\mathrm{I})}=\phi_{\mathrm{II}}-\phi_{\mathrm{I}}(5)$

eqn. (5) $(H \sim$ Henderson). In the special case of the cell (2) we obtain eqn. (6).

$\Delta \phi_{\mathrm{H}}=\frac{R T}{F} \frac{2 C_{1}\left(U_{\mathrm{Ba}}-U_{\mathrm{Cl}}\right)-C_{2}\left(U_{\mathrm{H}}-U_{\mathrm{Cl}}\right)}{2 C_{1}\left(2 U_{\mathrm{Ba}}+U_{\mathrm{Cl}}\right)-C_{2}\left(U_{\mathrm{H}}+U_{\mathrm{Cl}}\right)} \times$

$\ln \frac{2 C_{1}\left(U_{\mathrm{Cl}}+2 U_{\mathrm{Ba}}\right)}{C_{2}\left(U_{\mathrm{H}}+U_{\mathrm{Cl}}\right)}=\phi_{\text {r.h.s. }}-\phi_{\text {1.h.s. }}=\phi_{\mathrm{HCl}}-\phi_{\mathrm{BaCl}_{2}}$

The expression (5) may be transformed as follows: We introduce the ideal specific conductivity, eqn. (7), and the ideal transport number of the ith ion, eqn. (8), into eqn. (5). Hence, eqn. (9).

$\kappa^{0}=F^{2} \sum z_{\mathrm{i}}^{2} U_{\mathrm{i}} C_{\mathrm{i}}$

$t_{\mathrm{i}}^{0}=\frac{z_{\mathrm{i}}^{2} U_{\mathrm{i}} C_{\mathrm{i}}}{\sum z_{k}^{2} U_{k} C_{k}}$

$\Delta \phi_{\mathrm{H}}=-\frac{R T}{F} \cdot \frac{1}{\Delta \kappa^{0}} \cdot \Delta\left\{\kappa^{0} \Sigma \frac{t_{\mathrm{i}}^{0}}{z_{\mathrm{i}}}\right\} \ln \frac{\kappa^{0}(\mathrm{II})}{\kappa^{0}(\mathrm{I})}$

Compared with (5) eqn. (9) has the advantage of containing the experimentally determinable quantities $t_{\mathrm{i}}$ and $\kappa$ which also are defined at finite concentrations. By omission of the supercript zero, we can hope to be able to correct for concentration dependence of the conductivity and the transport numbers in a meaningful way.

In the case of two uni-uni valent electrolytes with one common ion (the anion, say) we express the two electrolytes as BA and DA, respectively, with $\mathrm{A}$ as common ion and we obtain eqns. (10) and (11),

$\Delta\left\{\kappa^{0} \Sigma \frac{t_{\mathrm{i}}^{0}}{z_{\mathrm{i}}}\right\}=F^{2}\left\{C(\mathrm{II}) U_{\mathrm{D}}-C(\mathrm{I}) U_{\mathrm{B}}-(\Delta C) U_{\mathrm{A}}\right\}$

$\Delta \kappa^{0}=F^{2}\left\{\mathrm{C}(\mathrm{II}) U_{\mathrm{D}}-\mathrm{C}(\mathrm{I}) U_{\mathrm{B}}+(\Delta C) U_{\mathrm{A}}\right\}$

where $\mathrm{C}(\mathrm{I})$ is the concentration of $\mathrm{BA}$ and $\mathrm{C}(\mathrm{II})$ the concentration of DA. For $\Delta C=0$ we have a LewisSargent cell. As would be expected eqn. (9) together with eqns. (10) and (11) passes over to eqn. (1) for $\Delta C=0$. 
Only total emf-values can be measured, however. The experimentally measured emf-values for the $\mathrm{BaCl}_{2} / \mathrm{HCl}$ cells are compared firstly to a simple Henderson emf, eqn. (12), where the last terms are

$\mathrm{EMF}_{\mathrm{H}}=\Delta \phi_{\mathrm{H}}-\frac{R T}{F} \ln \frac{C_{\mathrm{HCl}}}{2 C_{\mathrm{BaCl}_{2}}}$

the contributions due to the $\mathrm{Ag} / \mathrm{AgCl}$ electrodes in the ideal approximation. Secondly, we compare with an extended Henderson (EH) model similar to (12), but with $\Delta \phi_{\mathrm{EH}}$ calculated from the operational expression (9) using actual, experimental values for the conductivities and the transport numbers in the outer solutions. Thirdly, an extended Henderson "corrected" for activity coefficients (EHA) on the electrode potentials is also compared to experiments. The critical point is naturally the assumption regarding single ion activities. Strictly speaking, such an assumption would be superfluous, if the profiles $C_{\mathrm{BaCl}_{2}}(\mathrm{X})$ and $C_{\mathrm{HCl}}(\mathrm{X})$ were known throughout the zone and if $t_{\mathrm{H}^{+}}, t_{\mathrm{Ba}^{2}}+\ln a_{\mathrm{HCl}}$, and $\ln a_{\mathrm{BaCl}_{2}}$ were all known as functions of $C_{\mathrm{HCl}}$ and $C_{\mathrm{BaCl}_{2}}$. This is not our situation at present, so we are forced to make some assumptions. We try the different assumptions eqns. (13) and (14), where EHAI and

$\mathrm{EMF}_{\mathrm{EHAI}}=\Delta \phi_{\mathrm{EH}}-\frac{R T}{F} \ln \frac{y_{ \pm}(\mathrm{HCl}) C_{\mathrm{HCl}}}{y_{ \pm}\left(\mathrm{BaCl}_{2}\right) 2 C_{\mathrm{BaCl}_{2}}}$

$\mathrm{EMF}_{\mathrm{EHAII}}=\Delta \phi_{\mathrm{EH}}-\frac{R T}{F} \ln \frac{y_{ \pm}(\mathrm{HCl}) C_{\mathrm{HCl}}}{\sqrt{y_{ \pm}\left(\mathrm{BaCl}_{2}\right)} 2 C_{\mathrm{BaCl}_{2}}}$

EHAII correspond to the assumptions eqns. (15), and eqns. (16a) and (16b), respectively.

$a_{\mathrm{Cl}^{-}}=y_{ \pm} C_{\mathrm{Cl}^{-}}$(in $\mathrm{BaCl}_{2}$ and $\left.\mathrm{HCl}\right) \mathrm{EHA} \mathrm{I}$

$a_{\mathrm{Cl}^{-}}=y_{ \pm} C_{\mathrm{Cl}^{-}}($in $\mathrm{HCl})$

EHA II (16a)

$a_{\mathrm{Cl}^{-}}=\sqrt{y_{ \pm}} C_{\mathrm{Cl}^{-}}\left(\right.$in $\left.\mathrm{BaCl}_{2}\right)$

EHA II (16b)

Eqn. (15) is a pure ad hoc assumption, whereas eqn. (16) follows from $y_{ \pm}^{2}=y_{\mathrm{H}} y_{\mathrm{Cl}}$ and the Debye-Hückel relation $\ln y_{\mathrm{H}} / \ln y_{\mathrm{Cl}}=1$ in $\mathrm{HCl}$ which yields $y_{\mathrm{Cl}}=y_{ \pm}$ in $\mathrm{HCl}$. For $\mathrm{BaCl}_{2}$ we have $y_{ \pm}^{3}=y_{\mathrm{Ba}} y_{\mathrm{Cl}}^{2}$ and $\mathrm{ln}$ $y_{\mathrm{Ba}} / \ln y_{\mathrm{Cl}}=2^{2}$ which yields $y_{\mathrm{Cl}}=\sqrt{y_{ \pm}}$in $\mathrm{BaCl}_{2}$.

It should be stressed that the assumptions (15) and $(16 a-b)$ are of a conventional nature. Bates and Robinson ${ }^{15}$ have discussed another convention based on measurements on a mixed $\mathrm{KCl}$ $\mathrm{KNO}_{3}$ bridge and comparison with the Stokes and
Robinson hydration theory for activity coefficients. Their calculations are based on the assumption that the Henderson formula in its simple form is applicable for the evaluation of the junction potential across the mixed bridge (Ref. 15, eqn. (12)). Since the validity of the Henderson formula is one of the objects of inquiry in the present paper, the application of the Bates-Robinson conventional single ionic activities would be a circuitry.

An extensive study of the function of the salt bridge has been initiated by Sørensen ${ }^{16}$ and carried further in a recent paper by three of us. ${ }^{17}$ Further measurements are presently performed in our laboratory. One of the results is that a mixed $\mathrm{KCl}$ $\mathrm{KNO}_{3}$ bridge is inferior to a pure $\mathrm{KCl}$ bridge in eliminating the junction potential.

Last, but not least, the hydration theory of Stokes and Robinson has been exposed to severe criticism in a recent paper by Sørensen (Ref. 18, Appendix II). The ASPEV theory seems to give consistent ionic radii from second virial coefficients and also radii consistent with the modified BornFuoss-Boyd-Zwanzig theory of ionic mobilities. ${ }^{18,19}$ Measurements of activities of $\mathrm{HCl}$ and $\mathrm{BaCl}_{2}$ in $\mathrm{HCl}-\mathrm{BaCl}_{2}$ mixtures are presently carried out in our laboratory in order to elaborate the ASPEV theory to a point where reasonable estimates may be given for single ionic activities.

With the present confusion in the area of single ionic activities, however, we have preferred to use only very simple conventions here. This will facilitate the corrections to be made later, when more reliable single ionic activity coefficients can be defined through statistical mechanical solution theory and through a better understanding of the function of the salt bridge.

The experimental results are also compared to the model of Pleijel ${ }^{2}$ using mobilities at infinite dilution at $25^{\circ} \mathrm{C}$. Following the procedure of Pleijel for the $\mathrm{BaCl}_{2}^{(\mathrm{I})} \mid \mathrm{HCl}^{(\mathrm{II})}$ junction we observe first, that we have three different valences $(+2,+1$, $-1)$ distributed as $(+2,-1)$ in solution $I$ and $(+1$, -1 ) in solution II.

The dimensionless diffusion potential, eqn. (17), has to satisfy eqn. (18), where $h$ is defined as in eqn. (19).

$\Delta \phi^{\prime} \equiv \frac{F}{R T}\left(\phi_{\mathrm{II}}-\phi_{\mathrm{I}}\right)$

$\ln \frac{2(\lambda-1)}{\lambda-2}=\lambda \Delta \phi^{\prime}-\ln h$

Acta Chem. Scand. A 34 (1980) No. 7 
$h \equiv \frac{3 C_{\mathrm{BaCl}_{2}}}{2 C_{\mathrm{HCl}}}$

Eqn. (18) has for a given $\Delta \phi^{\prime}$ two different roots $\lambda_{1}$ and $\lambda_{2}$ which have also to fulfill the equation

$\frac{U_{\text {Ba }}}{3}\left(2-\lambda_{1}\right)\left(2-\lambda_{2}\right)-\frac{U_{\mathrm{H}}}{2}\left(1-\lambda_{1}\right)\left(1-\lambda_{2}\right)+$

$\frac{U_{\mathrm{Cl}}}{6}\left(1+\lambda_{1}\right)\left(1+\lambda_{2}\right)=0$

We have solved the system of eqns. (18) $-(20)$ in the following way. Eqn. (18) is written for $\lambda_{1}$ and for $\lambda_{2}$ and the two equations are divided by $\lambda_{1}$ and $\lambda_{2}$, respectively. Then the two equations are subtracted thus eliminating $\Delta \phi^{\prime}$.
$Z\left(\lambda_{1}, \lambda_{2}\right) \equiv$

$\frac{1}{\lambda_{1}}\left[\ln \frac{2\left(\lambda_{1}-1\right) h}{\lambda_{1}-2}\right]-\frac{1}{\lambda_{2}}\left[\ln \frac{2\left(\lambda_{2}-1\right) h}{\lambda_{2}-2}\right]=0$

Now, we choose a value of $\lambda_{2}$. From (20) we calculate the corresponding value of $\lambda_{1}$. Then we calculate $Z\left(\lambda_{1}, \lambda_{2}\right)$ which is generally different from zero. By scanning with $\lambda_{2}$ in the complex plane a pair $\left(\lambda_{1}, \lambda_{2}\right)$ can be found satisfying (20) and (21). Then $\Delta \phi^{\prime}$ is calculated from eqn. (18) for $\lambda=\lambda_{1}$, or $\lambda=\lambda_{2}$ (same result). The values of emf are calculated with ideal electrode contribution and with the two assumptions on activity coefficients used for the Henderson formula, and all calculations are compared to experiments.

Table 1. Mean values of measured emf's of cell (2) for fixed values of $C_{\mathrm{HCl}}=C_{2}$ and variable values of $C_{\mathrm{BaCl}_{2}}=C_{1} . \mathrm{SDM}=$ standard deviation of mean value. $N$ is the number of measurements. All values at $25.0 \pm 0.1^{\circ} \mathrm{C}$.

\begin{tabular}{|c|c|c|c|c|c|c|c|c|}
\hline$C_{1}$ & $\begin{array}{l}\operatorname{emf}\left(C_{2}=0.0001\right) \\
\pm \mathrm{SDM}\end{array}$ & $N$ & $\begin{array}{l}\operatorname{emf}\left(C_{2}=0.0010\right) \\
\pm \mathrm{SDM}\end{array}$ & $N$ & $\begin{array}{l}\operatorname{emf}\left(C_{2}=0.0100\right) \\
\pm \mathrm{SDM}\end{array}$ & $N$ & $\begin{array}{l}\operatorname{emf}\left(C_{2}=0.1000\right) \\
\pm \mathrm{SDM}\end{array}$ & $N$ \\
\hline 0.00050 & & & & & $-111.15 \pm 0.25$ & 2 & $-203.25 \pm 0.35$ & 2 \\
\hline 0.00100 & & & $-5.25 \pm 0.15$ & 2 & & & $-174.03 \pm 0.13$ & 3 \\
\hline 0.00141 & & & $-1.55 \pm 0.18$ & 2 & & & & \\
\hline 0.00200 & & & & & $-62.71 \pm 0.11$ & 2 & & \\
\hline 0.00208 & & & $8.36 \pm 0.16$ & 2 & & & & \\
\hline 0.00295 & & & & & $-49.43 \pm 0.08$ & 2 & & \\
\hline 0.00309 & & & $18.39 \pm 0.12$ & 3 & & & & \\
\hline 0.00316 & $69.78 \pm 0.69$ & 4 & & & & & & \\
\hline 0.00437 & & & & & $-37.12 \pm 0.08$ & 3 & & \\
\hline 0.00457 & & & $26.31 \pm 0.61$ & 3 & & & & \\
\hline 0.00500 & & & & & $-30.15 \pm 0.05$ & 2 & $-113.60 \pm 0.20$ & 2 \\
\hline 0.00562 & $81.37 \pm 0.87$ & 2 & & & & & & \\
\hline $\begin{array}{l}0.00646 \\
0.00676\end{array}$ & & & & & $-25.04 \pm 0.24$ & 3 & & \\
\hline $\begin{array}{l}0.00676 \\
0.00955\end{array}$ & & & $30.46 \pm 1.65$ & 2 & & & & \\
\hline 0.00955 & & & & & $-13.69 \pm 0.29$ & 3 & & \\
\hline 0.01000 & $92.01 \pm 0.25$ & 3 & $43.64 \pm 0.28$ & 10 & $-15.75 \pm 0.54$ & 2 & & \\
\hline 0.01413 & & & & & $-3.73 \pm 0.30$ & 3 & & \\
\hline 0.01778 & $101.42 \pm 0.39$ & 5 & $55.10 \pm 0.07$ & 3 & & & & \\
\hline 0.02084 & & & & & $5.42 \pm 0.09$ & 4 & & \\
\hline 0.03090 & & & & & $14.46 \pm 0.13$ & 4 & & \\
\hline 0.03162 & $111.48 \pm 0.55$ & 4 & $66.56 \pm 0.31$ & 5 & & & & \\
\hline 0.04571 & & & & & $22.79 \pm 0.52$ & 5 & & \\
\hline 0.05000 & & & & & & & $-32.65 \pm 0.05$ & 2 \\
\hline 0.05623 & $121.70 \pm 0.23$ & 4 & $77.35 \pm 0.11$ & 3 & & & & \\
\hline 0.06761 & & & & & $33.40 \pm 1.48$ & 4 & & \\
\hline 0.08250 & & & & & $36.53 \pm 0.03$ & 2 & & \\
\hline 0.10000 & $131.58 \pm 0.20$ & 4 & $87.17 \pm 0.27$ & 7 & $39.61 \pm 0.37$ & 8 & $-16.30 \pm 0.90$ & 2 \\
\hline 0.17780 & & & $97.45 \pm 0.23$ & 3 & $50.28 \pm 0.18$ & 5 & & \\
\hline 0.31620 & & & $107.07 \pm 0.40$ & 8 & $61.25 \pm 0.31$ & 3 & & \\
\hline 0.56230 & & & $116.41 \pm 0.30$ & 8 & $71.25 \pm 0.08$ & 3 & & \\
\hline 1.00000 & & & $126.03 \pm 0.30$ & 8 & $84.04 \pm 0.74$ & 2 & $40.55 \pm 0.15$ & 2 \\
\hline
\end{tabular}




\section{RESULTS AND DISCUSSION}

Table 1 shows the mean values of the measured emfs for cell (2) together with the standard deviation of mean value (SDM) for the concentration of $\mathrm{HCl}\left(C_{2}\right)$ fixed at the levels $0.0001,0.001,0.01$ and $0.1 \mathrm{~mol} / \mathrm{dm}^{3}$, respectively, and varying concentrations of $\mathrm{BaCl}_{2}\left(C_{1}\right)$.

The mean value $\bar{x}_{\mathbf{i}}$ is calculated by means of

$\bar{x}=\frac{1}{N} \sum_{\mathrm{i}=1}^{N} x_{\mathrm{i}}$

and the standard deviation of mean value

$\mathrm{SDM}=\left[\frac{1}{N(N-1)} \times \sum_{\mathrm{i}=1}^{N}\left(x_{\mathrm{i}}-\bar{x}\right)^{2}\right]^{1 / 2}$

with $x_{\mathrm{i}}=\mathrm{emf}$ (measured) and where $N$ is the number of measurements for each $C_{1} / C_{2}$-value.

In Fig. 3, a section of the measurements is shown in the decade $\log \left(C_{1} / C_{2}\right)$ from 1 to 2 and compared to calculations from the simple Henderson theory and from the Pleijel theory. Previously ${ }^{11}$ we have reported a more restricted set of measurements on the $\mathrm{BaCl}_{2} / \mathrm{HCl}$ system, leading to a rough picture of the emf $v s . \log \left(C_{1} / C_{2}\right)$ relationship. Fig. 3 shows a much more detailed picture which clearly expresses a hitherto unseen feature in this relationship: The split-up of the curves in such a way that each $C_{2}$-value defines its own curve.

We have fitted the data for each fixed $C_{2}$ with a least square polynomial in $\log \left(C_{1} / C_{2}\right)$. The procedure used to fit the significant polynomial was the method of orthogonal polynomials described earlier in a paper by one of us. ${ }^{12}$

Standard deviation of the polynomials (SDP) is a function of $p$, eqn. (22), with the greatest values of

$p \equiv \log \left(C_{1} / C_{2}\right)$

SDP at the terminal values of $p$.

The polynomials were fitted to the measurements with the results given in Table 2 .

The reason for the relatively large uncertainties on the polynomial given in eqn. (26) is that those measurements are the ones reported earlier ${ }^{11}$ with a spacing in $p$ considerably larger than for the polynomials given in eqns. (23), (24) and (25).

As seen from Fig. 3 it is not convenient to plot all the measured emf-values over the entire range of $p$-values. Therefore, on Fig. 4 we have plotted the

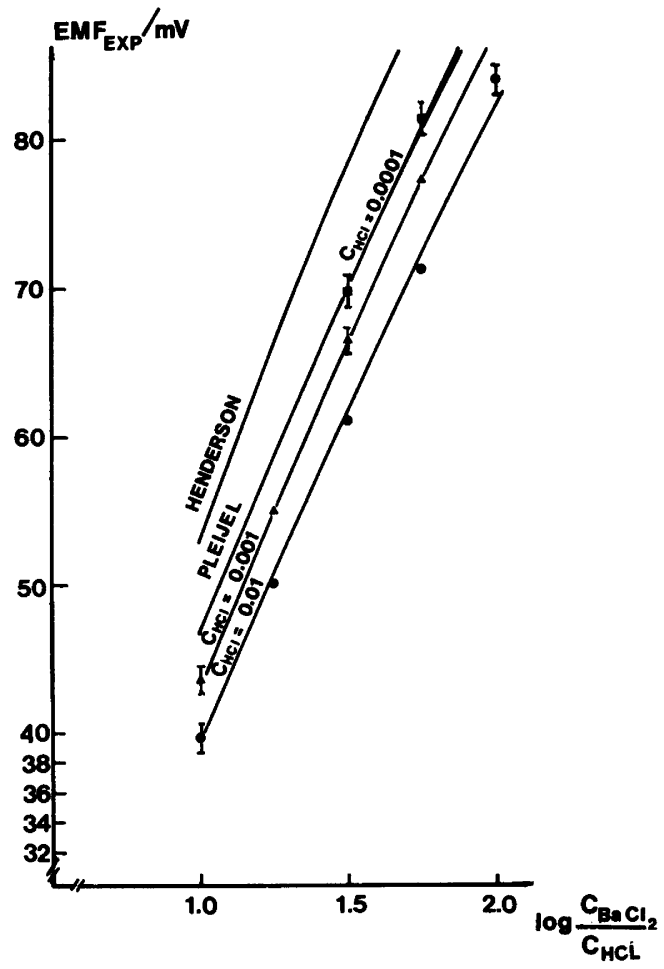

Fig. 3. Measured emf on cell (2) as function of log $\left(C_{1} / C_{2}\right)$ in the decade from 1 to 2 together with calculated emf-values from the simple Henderson model [eqns. (6) and (12)] and from the Pleijel model [eqns. (18)-(20)]. As clearly demonstrated the measured emf's are collected on three different curves determined by the three $C_{2}$ values 0.01 , 0.001 and 0.0001 , respectively.

Curve fit has been made by means of a least square polynomial in $\log \left(C_{1} / C_{2}\right)$. The three different $C_{2}$-values as well as the Henderson and the Pleijel models are indicated on the corresponding curves.

difference between the experimental values and the values calculated from the simple Henderson formula eqns. (6) and (12) as a function of $p$ for the four values of $C_{2}$. The mobilities used in calculating $\Delta \phi_{\mathrm{H}}$ have the ratios given in (27),

$\left(U_{\mathrm{H}}: U_{\mathrm{Ba}}: U_{\mathrm{Cl}}\right)=(349.81: 31.82: 76.35)$

determined from equivalent conductance at infinite dilution. ${ }^{15}$

Conversely, from Fig. 4, the data in (27) and the eqns. (6) and (12) it is possible to reproduce any 
Table 2. The results obtained when the polynomials (23)-(26) were fitted to the measurements.

$\operatorname{emf}($ polyno $)=1.344+48.285 p-1.642 p^{2}$

emf(polyno $)=-6.540+45.611 p+8.950 p^{2}-6.043 p^{3}+0.969 p^{4}$

emf(polyno $)=-13.267+61.011 p-9.492 p^{2}+1.451 p^{3}$

$\operatorname{emf}($ polyno $)=-15.268+63.884 p-7.810 p^{2}$

\begin{tabular}{lcccc}
\hline$C_{2} /\left(\mathrm{mol} / \mathrm{dm}^{3}\right)$ & 0.0001 & 0.001 & 0.01 & 0.1 \\
Polynomial & $(23)$ & $(24)$ & $(25)$ & $(26)$ \\
$p \in$ & {$[1.5 \mid 3.0]$} & {$[0.0 \mid 3.0]$} & {$[-1.3 \mid 2.0]$} & {$[-2.3 \mid 1.0]$} \\
Significance level & 0.05 & $<0.001$ & $<0.001$ & $<0.001$ \\
$p ;$ SDP $/ \mathrm{mV}$ & $1.5 ; 0.4$ & $0.0 ; 0.7$ & $-1.3 ; 0.9$ & $-2.3 ; 0.8$ \\
& $2.3 ; 0.2$ & $1.0 ; 0.3$ & $0.0 ; 0.2$ & $-0.5 ; 0.7$ \\
& $3.0 ; 0.4$ & $3.0 ; 0.4$ & $2.0 ; 0.9$ & $1.0 ; 0.9$ \\
\hline
\end{tabular}

value of emf at any given $p$-value and at any of the four $C_{2}$-values within the accuracy of the polynomial fits.

Roughly speaking, Fig. 4 shows; that experimental values are higher than $\mathrm{EMF}_{\mathrm{H}}$ for low $p$ values and lower for high $p$-values. The best fit is around $p=-0.3$, corresponding to $C_{\mathrm{BaCl}_{2}} / C_{\mathrm{HCl}}=$ 0.5 . Interestingly enough, this is exactly the condition of equal concentrations of the $\mathrm{Cl}^{-}$ion in the

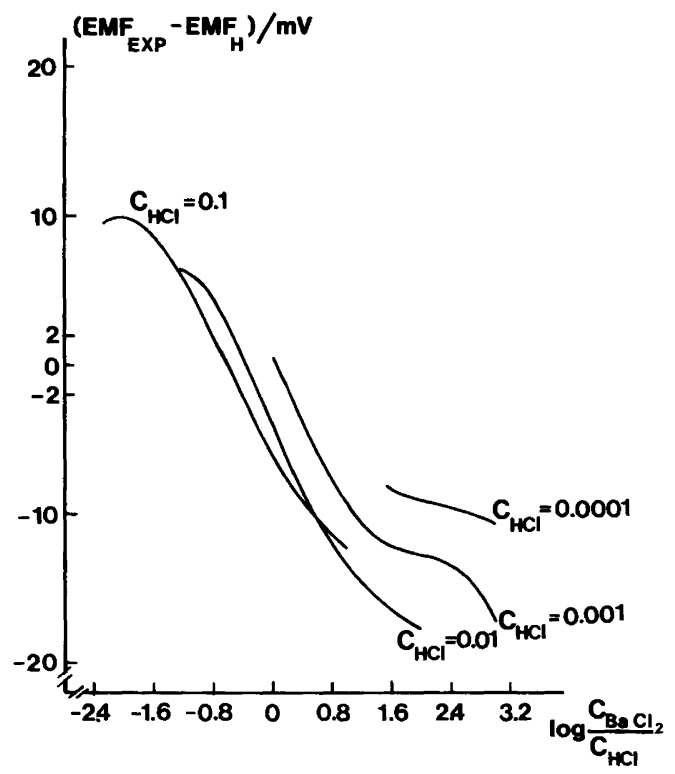

Fig. 4. Plot of differences between fitted experimental emf-values and emf-values calculated from the simple Henderson-model (eqns. (6) and (12)) as a function of $\log \left(C_{1} / C_{2}\right)$ for four values of $C_{2}$, i.e. $C_{2}=0.1,0.01,0.001$ and $0.0001 \mathrm{~mol} \mathrm{dm}^{-3}$.

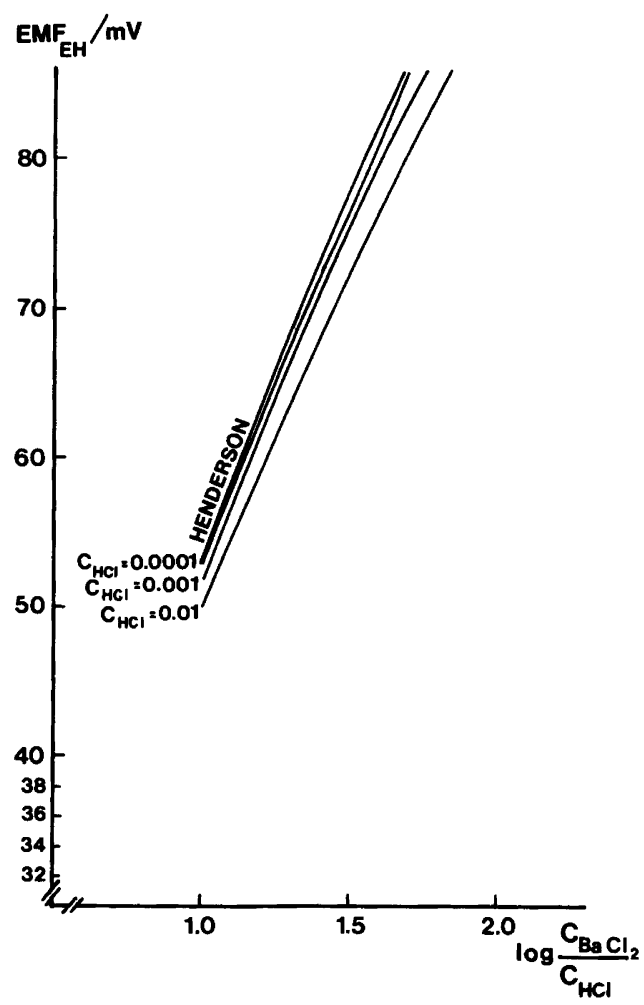

Fig. 5. The extended Henderson model (EH-model) plotted as a function of $\log \left(C_{1} / C_{2}\right)$ in the decade from 1 to 2 for three values of $C_{2}$, i.e. $C_{2}=0.01$, 0.001 and 0.0001 . As seen the EH-model leads to a split-up of the curves for different $C_{2}$-values. For comparison the simple Henderson model is also shown on the figure (indicated by HENDERSON). From this model only one emf can be calculated at a given $\log \left(C_{1} / C_{2}\right)$-value.

Acta Chem. Scand. A 34 (1980) No. 7 
external solutions and cell (2) represents, in this case, a modified Lewis-Sargent cell. The general fit of the Henderson formula is not better than within about $10 \mathrm{mV}$ too low $(p=-2)$ to about $18 \mathrm{mV}$ too high $(p=2)$. There is a clearcut separation between curves belonging to different values of $C_{\mathrm{HCl}}$. This separation ranges up to values of $9 \mathrm{mV}$.

In Fig. 5 the calculations using the extended Henderson formula (eqn. (9) with actual values of $\kappa(=c \Lambda), t_{\mathrm{Ba}}$ and $t_{\mathrm{H}}$ instead of values for infinite dilution) are shown in the decade from $p=1$ to $p=2$. We notice now, that there is a splitup of the curves for various values of $C_{\mathrm{HCl}}$. This stands in contrast to the case of the simple Henderson equation, also shown on Fig. 5 for comparison. By comparison with the experimental values on Fig. 3 in the same decade, we observe that the experimental split-up is larger than predicted by the EH-model.

Values of $\Lambda_{\mathrm{HCl}}, \Lambda_{\mathrm{BaCl}_{2}}, t_{\mathrm{H}}(\mathrm{HCl})$ and $t_{\mathrm{Ba}}\left(\mathrm{BaCl}_{2}\right)$ in pure solutions were taken from Landolt-Börnstein's tables ${ }^{20}$ and fitted with the polynomium program. ${ }^{12}$ We obtain eqns. (28) and (29) for the molar conductivities.

$\Lambda_{\mathrm{HCl}}=425.72-157.90 C_{2}^{1 / 2}+184.41 C_{2}-119.96 C_{2}^{3 / 2}$

$\left(C_{2}\right.$ from 0 to $1 \mathrm{~mol} / \mathrm{dm}^{3}$ )

$\Lambda_{\mathrm{BaCl}_{2}}=280.06-553.38 C_{1}^{1 / 2}+1610.36 C_{1}$

$-2910.26 C_{1}^{3 / 2}+2645.47 C_{1}^{2}-934.28 C_{1}^{5 / 2}$

( $C_{1}$ from 0 to $1 \mathrm{~mol} / \mathrm{dm}^{3}$ ).

For the transport numbers we have eqns. (30) and (31).

$t_{\mathrm{H}}=0.8210+0.0472 C_{2}^{1 / 2}-0.0456 C_{2}+0.01841 C_{2}^{3 / 2}$

$\left(C_{2}\right.$ from 0 to $1 \mathrm{~mol} / \mathrm{dm}^{3}$ )

$t_{\mathrm{Ba}}=0.4549-0.1660 C_{1}^{1 / 2}-0.0612 C_{1}+$

$+0.3271 C_{1}^{3 / 2}-0.1828 C_{1}^{2}$

( $C_{1}$ from 0 to $1 \mathrm{~mol} / \mathrm{dm}^{3}$ ).

In Fig. 6 we have plotted the experimental EMF's minus $\mathrm{EMF}_{\mathrm{EH}}$ in the entire $p$-range. It is evident by comparison with Fig. 4 that the deviations are more symmetric around zero than for the simple Henderson model. This is due to the correction of the coefficients of transport to the actual values instead of the use of mobilities of infinite dilution. We also

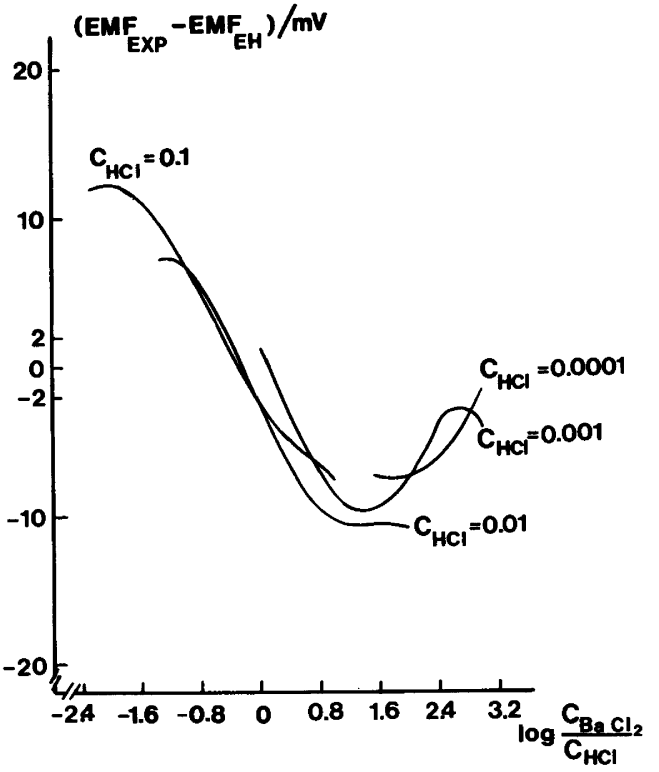

Fig. 6. Differences between fitted experimental emfvalues and emf-values calculated from the extended Henderson model (eqn. (9) with actual, experimental values for conductivity and transport numbers in the outer solutions) are plotted as a function of $\log$ $\left(C_{1} / C_{2}\right)$ for four values of $C_{2}$, i.e. $C_{2}=0.1,0.01$, 0.001 and $0.0001 \mathrm{~mol} \mathrm{dm}^{-3}$.

see that the split-up between the different $C_{2}$-levels are considerably less than on Fig. 4 (simple Henderson). This shows that the corrective split-up on Fig. 5 is in the right direction. The entire range of deviations is also "only" $23 \mathrm{mV}$ on Fig. 6 in comparison with $28 \mathrm{mV}$ on Fig. 4.

Using the activity assumption in eqn. (15) (EHAI) we obtain a considerably larger split-up than the experimental one, compare Figs. 7 and 3. Taking the difference between the experimental values and the EMF $_{\text {EHAI }}$ we observe (Fig. 8) that an asymmetry and a larger split-up are introduced over the entire $p$-range. The activity coefficients $\gamma_{ \pm}(\mathrm{HCl})$ are taken from Harned and Owen ${ }^{21}$ and transformed from molal to molar activity coefficients using the Table $12-1-1 \mathrm{~A}$, p. $725 .{ }^{21}$ The coefficients $\gamma_{ \pm}\left(\mathrm{BaCl}_{2}\right)$ are taken from various sources ${ }^{23-25}$ and transformed to molar activity coefficients using the density data for $\mathrm{BaCl}_{2}$ in Ref. 22 .

The data were treated by the computer program with the result: 


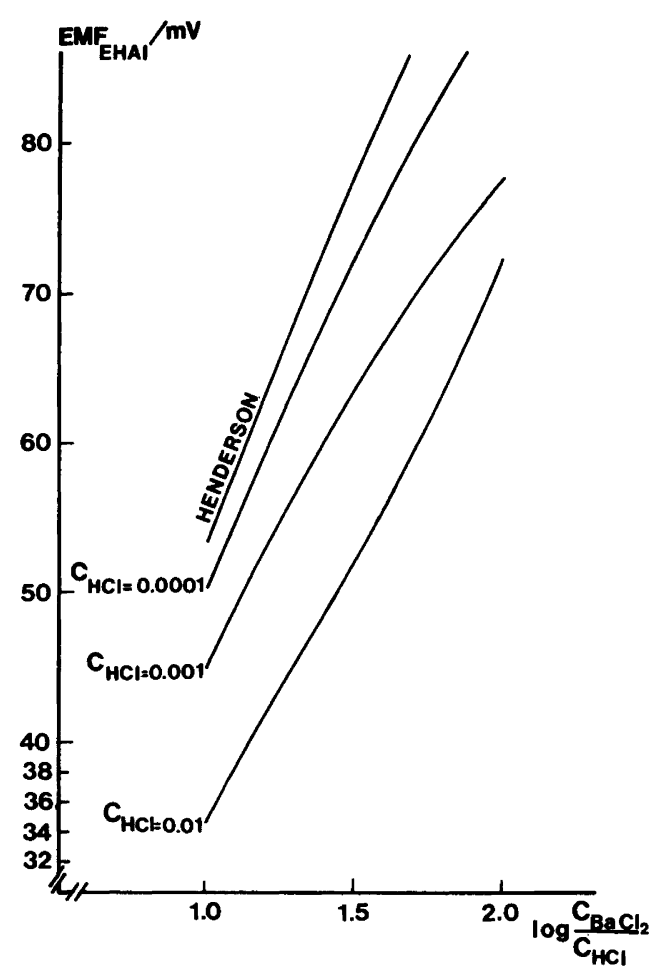

Fig. 7. Calculated emf-values from the EHAI model (eqn. (13)) as a function of $\log \left(C_{1} / C_{2}\right)$ in the decade from 1 to 2 . As seen the emf-values are split up into three different curves corresponding to the three $C_{2}$-values: $0.01,0.001$ and $0.0001 \mathrm{~mol}$ $\mathrm{dm}^{-3}$, respectively. The $C_{2}$-values are indicated on the curves. For comparison also the curve representing emf-values from the simple Henderson model is shown.

$\ln y_{ \pm}(\mathrm{HCl})=-1.169 C_{2}^{1 / 2}+1.750 C_{2}-1.002 C_{2}^{3 / 2}$

$\left(C_{2}\right.$ from 0 to $0.2 \mathrm{~mol} / \mathrm{dm}^{3}$ )

$\ln y_{ \pm}\left(\mathrm{BaCl}_{2}\right)=-2.271\left(3 C_{1}\right)^{1 / 2}+2.731\left(3 C_{1}\right)$

$-2.236\left(3 C_{1}\right)^{3 / 2}+1.181\left(3 C_{1}\right)^{2}-0.336\left(3 C_{1}\right)^{5 / 2}$

$\left(C_{1}\right.$ from 0 to $\left.1 \mathrm{~mol} / \mathrm{dm}^{3}\right)$.

The activity assumption in eqns. (16a) and (16b) (EHAII) turns out to be more appropiate. Fig. 9 shows the difference between the experimental values and $\mathrm{EMF}_{\mathrm{EHAII}}$ over the entire $p$-range. The deviations distribute themselves almost symmetrically around $0 \mathrm{mV}$ and the separations between different $C_{2}$-levels are quite small (up to $6 \mathrm{mV}$ ).

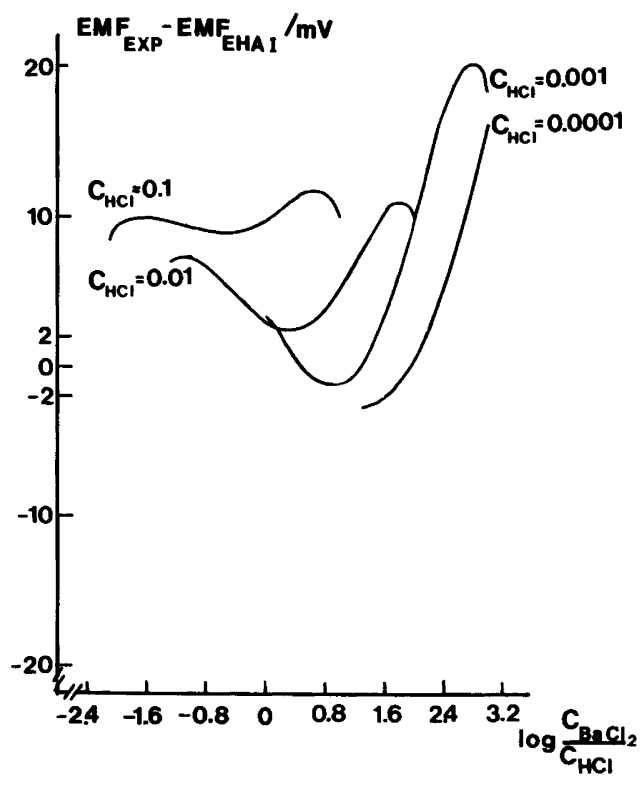

Fig. 8. Differences between fitted experimental emfvalues and emf-values calculated for the EHAImodel (eqn. (13)) as a function of $\log \left(C_{1} / C_{2}\right)$. Four different curves are obtained corresponding to four different $C_{2}$-values, i.e. $C_{2}=0.1,0.01$, 0.001 and $0.0001 \mathrm{~mol} \mathrm{dm}^{-3}$. $C_{2}$-values are indicated on the curves.

The entire range of values is within $14 \mathrm{mV}$. So this is the best fit we can obtain with primitive extensions of the Henderson formula.

Passing to the Pleijel-model a comparison with experiments is shown in Fig. 10 over the entire $p$ range. The deviations are asymmetrically distributed around zero due to the use of mobilities at infinite dilution (eqn. 27), but the range of values is only $14 \mathrm{mV}$ (like the EHAII model, but considerably less than the $28 \mathrm{mV}$ of the simple Henderson model).

Thus we may conclude that the uncorrected Pleijel model is better than the uncorrected Henderson model. This might be due to the fact that the concentration profiles move away from the continuous mixture profiles in the initial mixing zone when time passes. The profiles of stationary diffusion found in the Pleijel theory might be a better approximation to the quasi-stationary profiles of free diffusion. It is also obvious from Fig. 10 that the uncorrected Pleijel model fits the data quite satisfactorily when the $\mathrm{BaCl}_{2}$ as well as the $\mathrm{HCl}$ solution is dilute enough. This stands in contrast to the situation with 


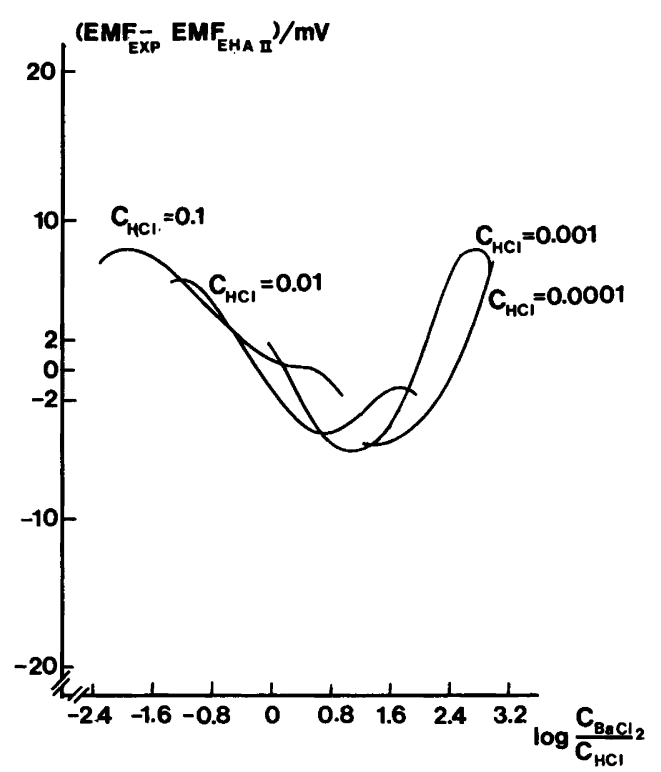

Fig. 9. Differences between fitted experimental emf-values and emf-values calculated from the EHAII-model (eqn. (14)) as a function of $\log$ $\left(C_{1} / C_{2}\right)$. Four different curves are obtained corresponding to four different $C_{2}$-values, i.e. $C_{2}=0.1$, $0.01,0.001$ and $0.0001 \mathrm{~mol} \mathrm{dm}^{-3} \cdot C_{2}$-values are indicated on the curves.

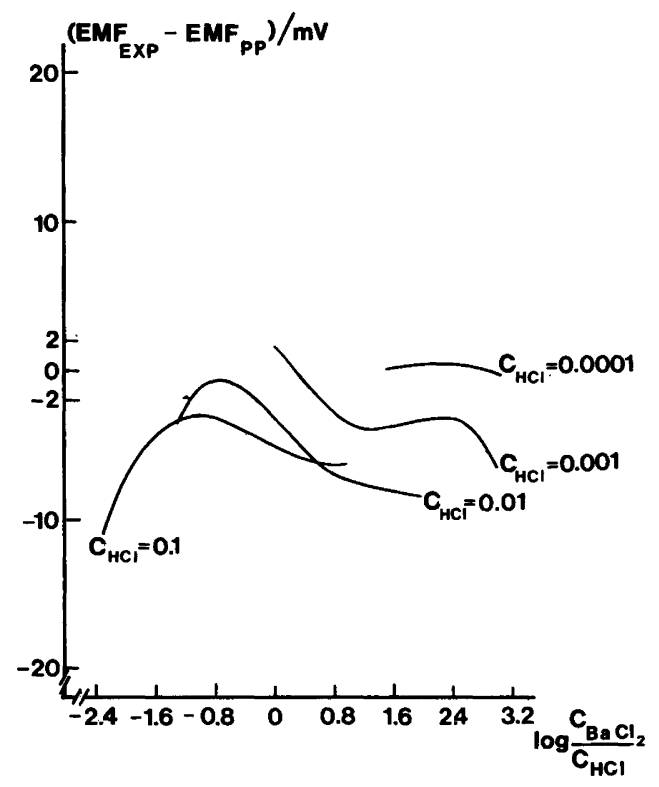

Fig. 10. Differences between fitted experimental emfvalues and emf-values calculated from the Pleijel model [eqns. (18)-(20)] as a function of $\log$ $\left(C_{1} / C_{2}\right)$. Four different curves are obtained corresponding to four different $C_{2}$-values indicated on the curves, i.e. $C_{2}=0.1,0.01,0.001$ and 0.0001 mol dm ${ }^{-3}$.

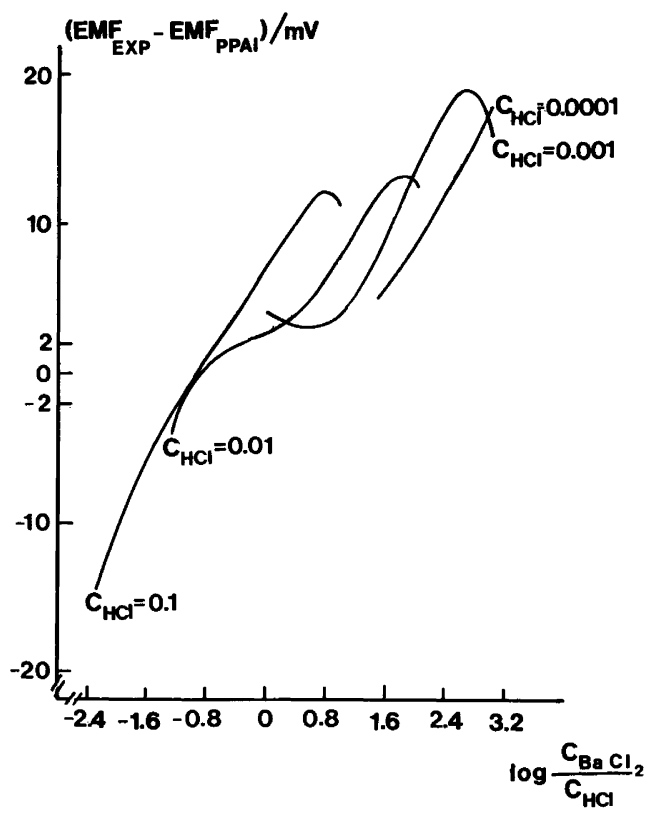

Fig. 11. Differences between fitted experimental and calculated emf-values as a function $\log \left(C_{1} / C_{2}\right)$. The calculated emf-values are obtained by means of the uncorrected Pleijel model in which the electrode potentials are corrected with activity coefficients according to eqn. (15). Four different curves are obtained corresponding to four different $C_{2}$-values indicated on the curves, i.e. $C_{2}=0.1$, $0.01,0.001$ and $0.0001 \mathrm{~mol} \mathrm{dm}^{-3} . C_{2}$-values are indicated on the curves.

the simple Henderson model (Fig. 4).

When the electrode potentials in the Pleijel model are corrected for activity coefficients according to eqn. (15) the final results look worse than the results from the uncorrected Pleijel model (compare Fig. 11 with Fig. 10). Similar effects were observed when the EHAI model (Fig. 8) was compared with the simple Henderson model (Fig. 4). However, using eqns. $(16 a-b)$ instead of eqn. (15) to correct for the electrode potentials in the Pleijel model (Fig. 12) we obtain a smaller separation between $C_{2}$-levels, but the entire range of values extends over $24 \mathrm{mV}$ which is considerably worse than the results obtained by using the simple Pleijel formula.

Comparing Fig. 12 with Fig. 9 (EHAII, the best model until now) the latter results seem puzzling. However, the "correction" for activity coefficients is only partial, since only the electrode potentials are corrected. Also, strictly speaking, the Pleijel model should have been corrected for varying transport coefficients, similar to the case for the EHAII mode. 


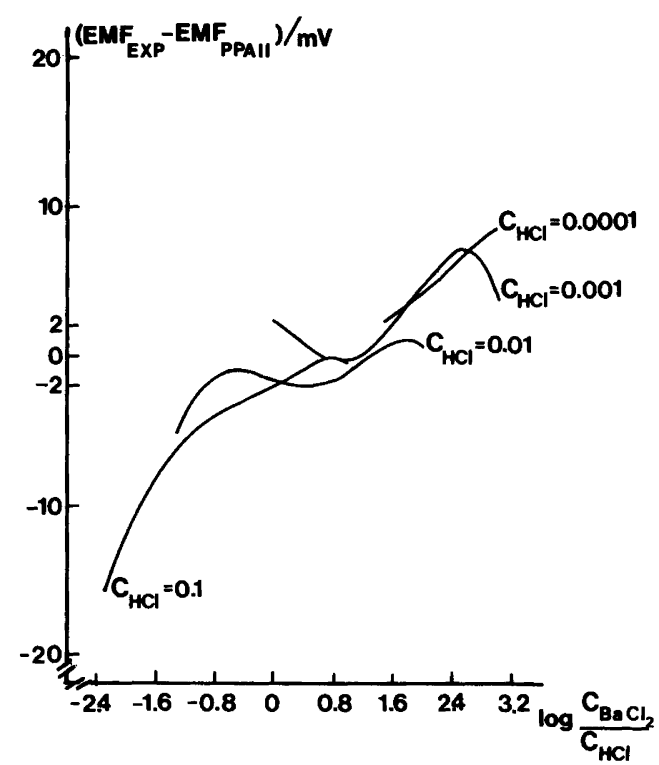

Fig. 12. Differences between fitted experimental and calculated emf-values as a function of $\log \left(C_{1} / C_{2}\right)$. The calculated emf-values are obtained by means of the uncorrected Pleijel model in which electrode potentials are corrected with activity coefficients according to eqns. (16a) and (16b). Four different curves are obtained corresponding to four different $C_{2}$-values indicated on the curves, i.e. $C_{2}=0.1$, $0.01,0.001$ and $0.0001 \mathrm{~mol} \mathrm{dm}^{-3} . C_{2}$-values are indicated on the curves.

In conclusion, on the basis of the present study, the EHAII model should be recommended for fast calculations, since the calculation according to Planck and Pleijel certainly is much more time consuming.

Acknowledgements. The authors wish to express their sincere thanks to civ.ing. Hans Georg Boye Nielsen, Radiometer $\mathrm{A} / \mathrm{S}$, for kindly delivering the $\mathrm{Ag} \mid \mathrm{AgCl}$-electrodes prepared in the thermalelectrolytic way. Thanks are also due to Lars Nygaard and Michael D. Pedersen for careful experimental and skilled technical assistance and their keen interest.

\section{REFERENCES}

1. Henderson, P. Z. Phys. Chem. 59 (1907) 118; $63(1908) 325$.

2. Pleijel, H. Z. Phys. Chem. 72 (1910) 1.
3. Planck, M. Ann. Physik 3, 39 (1890) 161; 40 (1890) 561.

4. Lewis, G. N. and Sargent, L. W. J. Am. Chem. Soc. 31 (1909) 363.

5. MacInnes, D. A. The Principles of Electrochemistry, Dover Publications, New York 1961 (1939).

6. Spiro, M. Electrochim. Acta 11 (1966) 569.

7. Bass, L. Trans. Faraday Soc. 60 (1964) 1914.

8. Goldman, D. E. J. Gen. Physiol. 27 (1943) 37.

9. Sørensen, T. S. and Jensen, K. F. J. Chem. Soc. Faraday Trans. 2, 71 (1975) 1805.

10. Zelman, D. A. J. Theor. Biol. 18 (1968) 396.

11. Østerberg, N. O., Jensen, J. B. and Sørensen, T. S. Acta Chem. Scand. A 32 (1978) 721

12. Sørensen, T. S. and Schack, P. In Hess, B. and Hemker, H. C., Eds., Analysis and Simulation of Biochemical Systems, North-Holland, Amsterdam 1972, p. 169.

13. Bates, R. G. Electrometric $p H$ Determinations, Wiley, New York 1954.

14. Ives, D. J. G. and Janz, G. J. Reference Electrodes, Academic, New York and London 1961.

15. Bates, R. G. and Robinson, R. A. Ion-Selective Electrodes, Pungor, E., Ed., Akadémiai Kiadó, Budapest 1978.

16. Sørensen, T. S. Studier over Fysisk-Kemiske Systemers Statik, Dynamik, Kinetik, Ph.D. Diss., Technical University of Denmark, Copenhagen 1973, Chapter III.

17. Østerberg, N. O., Sørensen, T. S. and Jensen, J. B. J. Electroanal. Chem. Interfacial Electrochem. In press.

18. Sørensen, T. S. Acta Chem. Scand. A 32 (1978) 571.

19. Sørensen, T. S. Acta Chem. Scand. A 33 (1979) 583.

20. Landolt-Börnstein, Zahlenwerte und Funktionen, II. Band, 7, Teil, Springer, Berlin-GöttingenHeidelberg 1960.

21. Harned, H. S. and Owen, B. B. The Physical Chemistry of Electrolytic Solutions, Reinhold, New York 1963.

22. Handbook of Chemistry and Physics, 50th Ed., The Chemical Rubber Co., Cleveland 1969.

23. Robinson, R. A. J. Am. Chem. Soc. 59 (1937) 84.

24. Tippetts, E. A. and Newton, R. F. J. Am. Chem. Soc. 56 (1934) 1675.

25. Ardizzone, S., Longhi, P., Mussini, T. and Rondinini, S. J. Chem. Thermodyn. 8 (1976) 557.

Received March 4, 1980. 